\title{
Experimental investigation on evaporation dynamics of sessile ethanol droplets on a heated substrate
}

\author{
Shuang Yea ${ }^{\mathrm{a}}$, Li Zhang ${ }^{\mathrm{b}}$, Chun-Mei Wu ${ }^{\mathrm{a}}$, You-Rong $\mathrm{Li}^{\mathrm{a}, *}$, Qiu-Sheng Liu ${ }^{\mathrm{c}}$ \\ ${ }^{a}$ Key Laboratory of Low-grade Energy Utilization Technologies and Systems of Ministry of Education, College of Energy and Power Engineering, Chongqing \\ University, Chongqing 400044, China \\ b Chongqing City Management College, Chongqing 401331, China \\ ${ }^{\mathrm{c}}$ Key Laboratory of Microgravity (National Microgravity Laboratory), Institute of Mechanics, Chinese Academy of Sciences, Beijing, 100190, China
}

\section{A R T I C L E I N F O}

\section{Article history:}

Received 17 April 2020

Revised 14 August 2020

Accepted 15 August 2020

Available online 26 August 2020

\section{Keywords:}

Experimental investigation

Sessile droplet

Evaporation

Thermal patterns

Heated substrate

\begin{abstract}
A B S T R A C T
In order to understand the evolution of thermal patterns and the effect of the thermal convection in the sessile droplet on the evaporation processes, a series of experimental investigations on evaporation dynamics of sessile ethanol droplets on a heated substrate were carried out based on the steady state evaporation method. The contact radius of the droplet on the substrate is fixed at $2.5 \mathrm{~mm}$ and the droplet height varies from 0.4 to $1.2 \mathrm{~mm}$. Results indicate that with the decrease of the droplet height, the temperature distribution near the droplet center becomes nonuniform, and hydrothermal waves and BénardMarangoni cells appear in sequence. The effect of liquid supplementation on the thermal patterns can be neglected. With the raise of the substrate temperature, both the wave number of the thermal waves and the frequency of the temperature fluctuation near the contact line increase. Moreover, the evaporation rate decreases first, and then increases with the decrease of the droplet height. The enhancing thermal convection in the droplet can promote the evaporation process at a high substrate temperature.
\end{abstract}

(c) 2020 Elsevier Ltd. All rights reserved.

\section{Introduction}

Since the droplet evaporation on a solid substrate is highly prevalent in daily life and industrial production, such as inject printing [1,2], spray cooling [3,4] and surface coating [5,6] etc., it is important to understand the evaporation dynamics of the sessile droplet. In recent years, many researchers have investigated the effects of different factors on the evaporation process of the sessile droplets. Among them, the interaction between thermal convection and droplet evaporation dynamics has become an increasingly popular research topic.

In the evaporation process of a sessile droplet, the coexistence of the tangential temperature gradient at the gas-liquid interface and the normal temperature gradient inside the droplet will induce various thermal convections, such as, the thermocapillary flow and buoyancy convection. These thermal convections can not only affect the deposition of particles in the droplet $[7,8]$, but also change the energy transfer during evaporation $[9,10]$. On the other hand, the intense and flow direction of these thermal convections are influenced by liquid properties [11,12], droplet geometry [13,14], and other factors that associated with the sessile droplet evaporation.

\footnotetext{
* Corresponding author.

E-mail address: liyourong@cqu.edu.cn (Y.-R. Li).
}

Actually, some thermal convections may destabilize when the intensity is large enough. At this time, a variety of thermal patterns associated with the thermal convections can be observed on the evaporating droplet surface through infrared image technology. The researches on the thermal patterns during sessile droplet evaporation are very rich, a lot of reports on the influences of liquid volatilities and droplet geometric structure on the thermal patterns were published.

For liquids with the different volatilities, the thermal patterns are quite different. Sefiane et al. [11] and Gleason et al. [15] experimentally studied the evaporation process of sessile water droplet, and the infrared images showed that there is no thermal pattern on the droplet surface. However, in the works of Josyula et al. [14] and Chen et al. [16], the thermal pattern with the stable twin vortices has been observed on the gas-liquid interface of water droplet that evaporates on a heated substrate. In general, as water is a non-volatile liquid, the thermal pattern on the droplet surface is not complicated when water evaporates on a solid substrate. However, for the evaporation of volatile sessile droplet, the thermal convection in the droplet will destabilize, and the thermal patterns on the droplet surface become more complicated. For the first time, Sefiane et al. [11] observed the thermal pattern on the evaporating surface of sessile ethanol and methanol droplets, and considered it as the hydrothermal waves (HTWs). Prior to that, 


\begin{tabular}{|c|c|}
\hline \multicolumn{2}{|c|}{ Nomenclature } \\
\hline$c_{\mathrm{p}}$ & specific heat capacity, $\mathrm{J} /(\mathrm{kg} \cdot \mathrm{K})$ \\
\hline$E_{\mathrm{V}}$ & evaporation rate, $\mu \mathrm{l} / \mathrm{min}$ \\
\hline$h$ & droplet height, mm \\
\hline$H$ & relative humidity, - \\
\hline$L_{\mathrm{V}}$ & vaporization heat, $\mathrm{kJ} / \mathrm{kg}$ \\
\hline$M a$ & Marangoni number, - \\
\hline$r$ & radial position, mm \\
\hline$R$ & radius, $\mathrm{mm}$ \\
\hline$t$ & time, $s$ \\
\hline$T$ & temperature, ${ }^{\circ} \mathrm{C}$ \\
\hline \multicolumn{2}{|c|}{ Greek symbols } \\
\hline$\alpha$ & thermal diffusivity, $\mathrm{m}^{2} / \mathrm{s}$ \\
\hline$\beta$ & thermal expansion coefficient, $1 / \mathrm{K}$ \\
\hline$\varepsilon$ & emissivity \\
\hline$\lambda$ & thermal conductivity, $\mathrm{W} /(\mathrm{m} \cdot \mathrm{K})$ \\
\hline$\mu$ & dynamic viscosity, mPa.s \\
\hline$\rho$ & density, $\mathrm{kg} / \mathrm{m}^{3}$ \\
\hline$\sigma_{\mathrm{T}}$ & temperature coefficient of surface tension, $\mathrm{N} /(\mathrm{m} \cdot \mathrm{K})$ \\
\hline \multicolumn{2}{|c|}{ Subscripts } \\
\hline 0 & initial value \\
\hline a & ambient \\
\hline ap & droplet apex \\
\hline $\mathrm{F}$ & full lifetime of droplet evaporation \\
\hline $\mathrm{h}$ & tangential value \\
\hline $\mathrm{T}$ & temperature coefficient \\
\hline $\mathrm{V}$ & normal value \\
\hline $\mathrm{W}$ & substrate \\
\hline
\end{tabular}

this thermal pattern was found to appear only in thin liquid layers with a tangential temperature gradient. The HTWs is a complicated thermal pattern which is characterized by the alternating temperature stripes of cold and warm propagating along a direction. Moreover, they also found that the convection cells rather than the HTWs appear on the evaporation surface of FC-72 droplet. It indicates that the thermal patterns may be very different for different volatile sessile droplets. However, Semenov et al. [17] believed that the HTWs on the evaporation surface of the ethanol droplet are actually the Bénard-Marangoni (B-M) convection cells. Brutin et al. [18] experimentally investigated the thermal patterns on the evaporation surface of ethanol and methanol droplets. They found that the cells on ethanol surface are more stable and the number of the cells is less than thoes on methanol droplet surface although the thermal patterns are similar (i.e., both of them are convection cells). In addition, the number of the cells on ethanol droplet surface increases with the raise of the substrate temperature. Furthermore, Sefiane et al. [19] innovatively studied the liquid-solid interface temperature and heat flux by using infrared imaging technology, and pointed out that the thermal patterns on the evaporation surface are actually bulk waves. As a result, the thermal patterns can affect the temperature distribution and heat flux on the liquid-solid interface, and change the evaporation rate. Similar conclusions are supported by Mahmud and MacDonald [9], Ghasemi and Ward [10], and Ward and Duan [20]. However, the effect of the thermal pattern on evaporation rate is rarely discussed in these reports.

In fact, there are several different modes during natural sessile droplet evaporation process. In 1977, Picnett and Bexon [21] presented two evaporation modes, they are constant contact radius (CCR) mode and constant contact angle (CCA) mode. As the name implies, CCR mode means that the contact radius remains constant while the contact angle decreases during evaporation; CCA mode refers to that the contact angle remains constant and the contact radius decreases. Nowadays, it is more widely recognized that there are four evaporation modes: CCR mode, CCA mode, mixed mode [22,23] and stick-slip mode [24]. Regardless of the evaporation mode, the geometric shape of droplet will change during the evaporation, which has a significant influence on the thermal convection inside the droplet and the evolution of the thermal patterns on the evaporation surface. Lu et al. [25] performed a numerical simulation on the internal flow of sessile water droplet evaporating on a heated substrate. They found that with the decrease of the contact angle, the internal flow evolves from a counterclockwise circulation flow into multiple circulation flows, and the multicellular structure should correspond to Bénard convection. Bouchenna et al. [26] used a quasi-steady-state method to investigate the thermocapillary flow in a sessile water droplet at CCR mode. Results showed that the internal flow direction changes with the decrease of the contact angle when water droplet evaporates on a PTFE substrate. Similar conclusion was also obtained by Chen et al. [13]. Considering that the change of droplet geometry will affect the observation on the thermal patterns, Zhong and Duan [27] adopted a steady state evaporation method to investigate the thermal pattern during the ethanol evaporation process. The results revealed that the HTWs also appear on the ethanol surface under steady state evaporation. Moreover, the higher the substrate temperature is, the larger the temperature oscillatory of the HTWs is. Using infrared imaging technology and adopting the steady-state evaporation method, Ye et al. [28] observed the evolution of the thermal pattern during the evaporation of sessile isopropanol droplets. It was found that several thermal patterns appear sequentially on the droplet surface with the decrease of the droplet height, including HTWs and petal-like patterns, etc. Wang and Shi [29] experimentally studied the transition of thermal patterns during the evaporation of sessile methanol and R113 droplets at CCR mode. The results showed that the HTWs and Bénard-Marangoni cells appear on the droplet surface with the decrease of the contact angle. Recently, Ye et al. [30]. investigated the evaporation of water and ethanol droplet under pure vapor environment at low pressures. The gear-like pattern and the cellular flow were found on the droplet surface with the decrease of the droplet height.

Previous researches have shown that the volatile droplets present complex thermal patterns on the evaporation surface, and the change of the droplet geometry has a significant effect on the thermal patterns. Furthermore, some researchers have reported that the thermal patterns can affect the evaporation rate of the sessile droplet. However, very few reports focus on the promotion action of the thermal convection on the evaporation rate. In present work, we observed the evolution of thermal patterns on the evaporating surface of ethanol droplets and revealed the promotion mechanism of the thermal convection on the evaporation rate by using the steady-state evaporation method and infrared image technology.

\section{Experimental apparatus}

The experimental apparatus for steady state evaporation of the sessile droplet is shown in Fig. 1. In order to prevent the influence of air convection on the droplet evaporation, the substrate is installed inside a stainless-steel chamber. Since the infrared camera is mounted vertically above the substrate, the top side of the chamber adopts a germanium infrared window to ensure that the infrared image camera (FLIR SC325, resolution: $320 \times 240$, the field of view: $8 \times 6 \mathrm{~mm}$, thermal sensitivity: $0.05{ }^{\circ} \mathrm{C}$ ) can be used to observe the droplet surface temperature. Two quartz glass windows are mounted on the left and right sides to facilitate the CMOS camera (PixeLINK, PL-B771, resolution: $800 \times 600$ ) to monitor the 


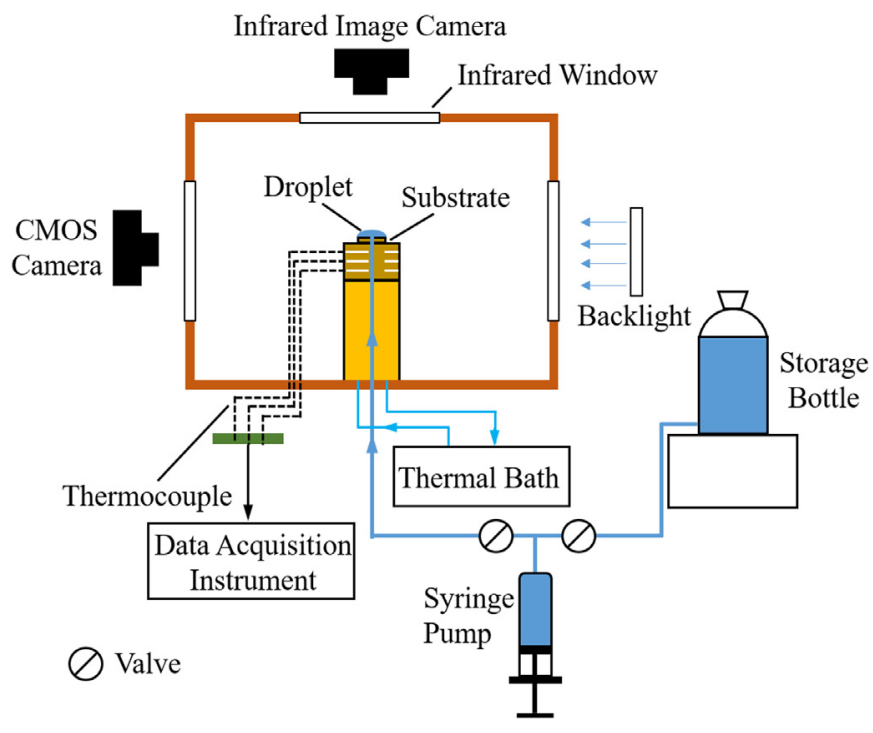

Fig. 1. The schematics of the experimental apparatus.

Table 1

Physical properties of ethanol at $25^{\circ} \mathrm{C}$ and $1 \mathrm{~atm}[18]$.

\begin{tabular}{lll}
\hline Parameter & Unit & Value \\
\hline B oiling point, $T_{\mathrm{b}}$ & ${ }^{\circ} \mathrm{C}$ & 78.0 \\
Density, $\rho$ & $\mathrm{kg} / \mathrm{m}^{3}$ & 789 \\
Dynamic viscosity, $\mu$ & $\mathrm{mPa} \cdot \mathrm{s}$ & 1.095 \\
Vaporization heat, $L_{\mathrm{V}}$ & $\mathrm{kJ} / \mathrm{kg}^{1}$ & 923 \\
Specific heat capacity, $c_{\mathrm{p}}$ & $\mathrm{J} /(\mathrm{kg} \cdot \mathrm{K})$ & 2845 \\
Thermal conductivity, $\lambda$ & $\mathrm{W} /(\mathrm{m} \cdot \mathrm{K})$ & 0.140 \\
Thermal expansion coefficient, $\beta$ & $1 / \mathrm{K}$ & $1.08 \times 10^{-3}$ \\
Surface tension temperature coefficient, $\sigma_{\mathrm{T}}$ & $\mathrm{N} /(\mathrm{m} \cdot \mathrm{K})$ & $0.83 \times 10^{-4}$ \\
Emissivity, $\varepsilon$ & - & $0.92[33]$ \\
\hline
\end{tabular}

droplet side view. The front of the chamber is connected to the atmosphere to avoid the vapor accumulation in the chamber. Thus, the influence of vapor accumulation on droplet evaporation and data measurement is avoided. The storage bottle is used to place ethanol in advance. The ethanol is provided by Chongqing Chuandong Co. Ltd., and the purity is higher than $99.7 \%$. Table 1 shows the physical properties of ethanol at $25^{\circ} \mathrm{C}$.

A syringe pump (KDS 200 series) is connected to the storage bottle and the substrate. The substrate in present work is the same with the previous one [28]. Fig. 2 shows the side view and the infrared image of the small substrate. The substrate is made of copper, and the radius and thickness of the small substrate are $R=2.5 \pm 0.05 \mathrm{~mm}$ and $\delta=0.5 \pm 0.05 \mathrm{~mm}$, respectively. The surface roughness of the small substrate is $0.8 \mu \mathrm{m}$. The hole for replenishing experimental liquid is located in the center of the small substrate, and its radius is $0.3 \pm 0.05 \mathrm{~mm}$. The substrate tempera- ture is maintained by a thermostatic water bath (Shanghai Qiqian Electronic Technology Co. Ltd, DC-2006, temperature ranges from $-20{ }^{\circ} \mathrm{C}$ to $100{ }^{\circ} \mathrm{C}$, and the temperature control accuracy is \pm 0.05 $\left.{ }^{\circ} \mathrm{C}\right)$. The substrate temperature is monitored by $12 \mathrm{~K}$-type thermocouples. When the set temperature is reached, the syringe pump is used to inject liquid onto the substrate to form droplets. It is worth mentioning that, in order to avoid the influence of the temperature of the injection liquid on the thermal pattern, before entering the droplet, the injection liquid will be sufficiently heated to make its temperature equal to the substrate temperature.

During the liquid injection process, the droplet side view is monitored in real time by the CMOS camera. At the same time, the side view obtained by the CMOS camera can be analyzed by an open source software Image (Image Processing and Analysis in Java) to obtain the height of the droplet [31]. By continuously adjusting the injection rate of the syringe pump, a droplet with a predetermined height is formed on the substrate. After that, stop adjusting the injection rate. If the droplet height changes no more than $45 \mu \mathrm{m}$ within $15 \mathrm{~min}$, the evaporation is considered to be stable. At this time, the evaporation rate is equal to the injection rate of the syringe pump. When the droplet evaporates to a stable state, the thermal pattern of droplet surface is recorded by infrared image camera.

\section{Experimental results and discussion}

In present work, the contact radius $R$ is equal to the radius of small substrate, i.e. $R=2.5 \mathrm{~mm}$. Change the droplet geometry only by adjusting the droplet height. Furthermore, for all experiments, the ambient temperature is controlled at $T_{\mathrm{a}}=25.0 \pm 1.0{ }^{\circ} \mathrm{C}$, and the relative humidity is $H=65 \% \pm 5 \%$.

To show the influence of droplet geometry on the thermal patterns, the side view and surface infrared images of the ethanol droplet at $T_{\mathrm{w}}=45{ }^{\circ} \mathrm{C}$ are shown in Fig. 3. The droplet height $h$ is defined as the distance from the small substrate to the droplet apex. As shown in Fig. 3(a), when $h=1.2 \mathrm{~mm}$, most of the surface has no obvious temperature fluctuation, while the temperature fluctuation at the top of droplet is ununiform. Actually, for these cases that the droplet height $h$ is higher than $1.2 \mathrm{~mm}$, the surface temperature distribution is similar to that at $h=1.2 \mathrm{~mm}$. After reducing the droplet height by adjusting the injection rate of the syringe pump, it is found that some striated thermal patterns begin to appear in the region near the three-phase contact line. With the further decrease of the droplet height, the region occupied by the striated thermal patterns extends gradually. When the droplet height is decreased to $0.8 \mathrm{~mm}$, the thermal pattern seems to be some curved temperature stripes near the contact line rotating longitudinally, as shown in Fig. 3(b). The rotating directions are marked by arrows in Fig. 3(b). Compared with the previous work [28] and the reports from Sefiane et al. [11], it is found that the characteristics of this thermal pattern are very similar to those of the HTWs. Therefore, it is believed that this thermal pattern should
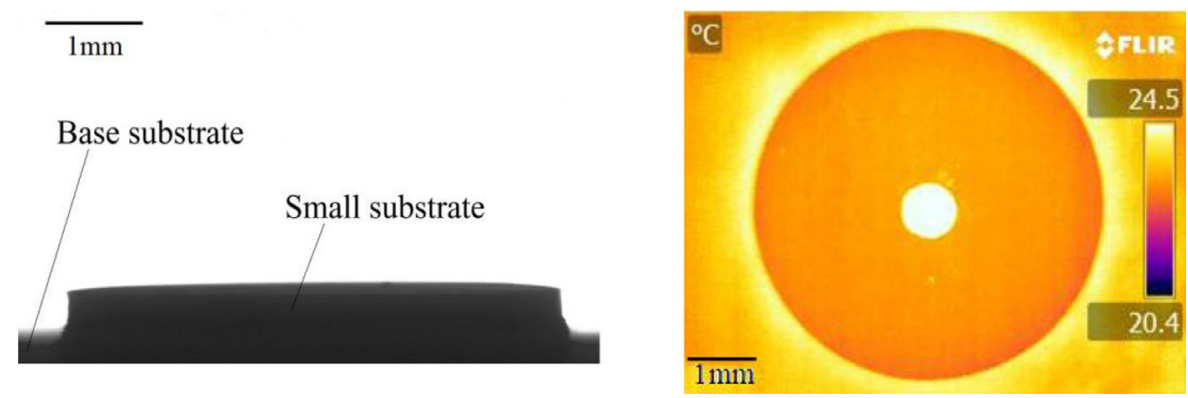

Fig. 2. The side view (left) and surface infrared imaging map (right) of the small substrate. 


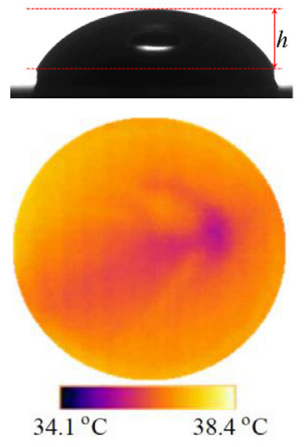

(a) $h=1.2 \mathrm{~mm}$

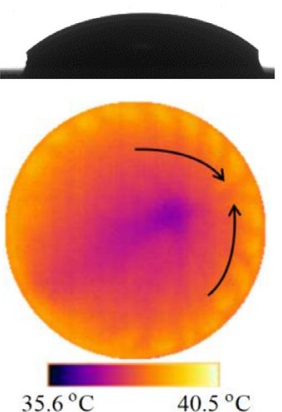

(b) $h=0.8 \mathrm{~mm}$

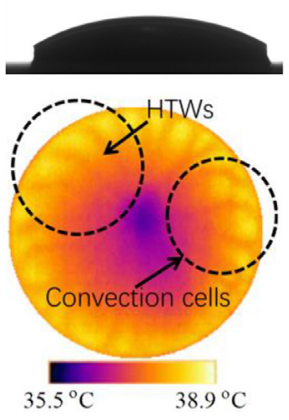

(c) $h=0.6 \mathrm{~mm}$

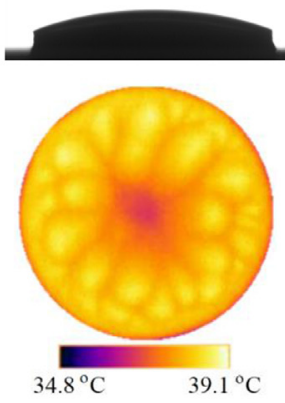

(d) $h=0.4 \mathrm{~mm}$

Fig. 3. The side view and surface infrared images of ethanol droplet at $T_{\mathrm{w}}=45^{\circ} \mathrm{C}$.

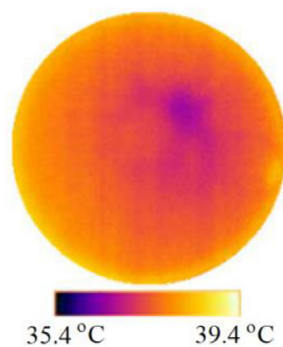

(a) $t / t_{\mathrm{F}} \approx 0.06$

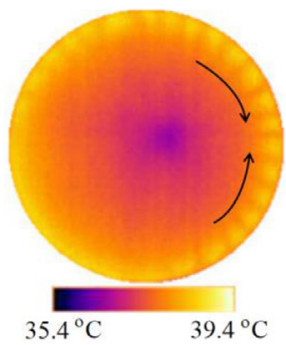

(b) $t / t_{\mathrm{F}} \approx 0.38$

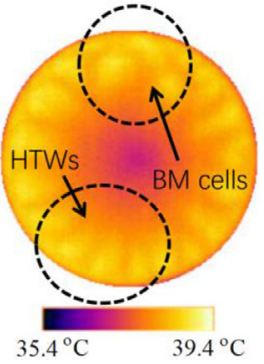

(c) $t / t_{\mathrm{F}} \approx 0.68$

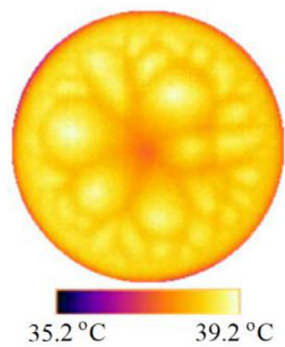

(d) $t / t_{\mathrm{F}} \approx 0.91$

Fig. 4. The surface infrared images of ethanol droplet without the liquid supplement.

be the HTWs. As displayed in Fig. 3(c), when the droplet height is decreased to $0.6 \mathrm{~mm}$, the HTWs still exist on the droplet surface. However, some convection cells (marked by the 'Convection cells' in Fig. 3(c)) generate near the contact line. Notably, at this time, these cells are first generated in the region near the contact line. Then, it will be squeezed by the new cells. After that, the original cells become smaller and continue to shrink toward the droplet center. Eventually, they disappear near the droplet center. When the drop height continues to decrease, as shown in Fig. 3(d), more and more convection cells appear. The thermal pattern at $h=0.4 \mathrm{~mm}$ is different from the situation that the convection cells first appear at $h=0.6 \mathrm{~mm}$. In addition to the continuous appearance of convection cells near the contact line, some cells also generate at the droplet center. Once the cell generates at the droplet center, it will become large. At the same time, the cell will move toward the contact line. Then, the big cell will split into two or more small cells in the contact line region. Finally, like the cells generated in the contact line region, they will shrink toward the droplet center, and disappear near the droplet center. These thermal patterns at $h=0.6 \mathrm{~mm}$ and $0.4 \mathrm{~mm}$ are very similar to those reported by Wang and Shi [29]. However, they studied the thermal model during transient evaporation of the methanol droplets and attributed this thermal pattern to the Bénard-Marangoni cells.

In the steady-state evaporation, the working liquid is supplied from the bottom of the droplet to maintain the shape of the droplet. Therefore, the effect of the liquid supplement on the thermal pattern must be carefully considered. As a comparative experiment, an ethanol droplet is formed on the small substrate with $T_{\mathrm{w}}=45{ }^{\circ} \mathrm{C}$ by using the syringe pump. It should be noted that the initial droplet height is higher than $1.2 \mathrm{~mm}$. After the droplet is formed, syringe pump is turned off to make the droplet evaporate naturally until it almost completely evaporated. Fig. 4 shows the thermal pattern evolution of the ethanol droplet without liquid supplement, where $t$ stands for the evaporating time, and $t_{\mathrm{F}}$ stands for the full lifetime of droplet evaporation. It is seen from Fig. 4(a) that when $t / t_{\mathrm{F}} \approx 0.06$, i.e. at the initial stage of the droplet evaporation, the surface temperature distribution is very similar to that in Fig. 3(a). When $t / t_{\mathrm{F}}$ is increased to about 0.38, the HTWs appear on the droplet surface, and their rotating directions are marked by arrows in Fig. 4(b). After that, when $t / t_{\mathrm{F}} \approx 0.68$, the droplet height decreases further as the evaporation continues, the thermal patterns show that the HTWs and Bénard-Marangoni cells coexist (the HTWs and B-M cells are marked by dash circle in Fig. 4(c)). Finally, when $t / t_{\mathrm{F}} \approx 0.91$, the droplet height is very low. Therefore, the thermal pattern is Bénard-Marangoni cells. In this case, the appearance and the movement of the cells are similar to those at $h=0.4 \mathrm{~mm}$ in the steady state evaporation. By comparing Figs. 3 and 4, it can be found that the effect of liquid supplementation on the thermal patterns is negligible.

According to Figs. 3 and 4, the variation of the thermal patterns with the droplet height is very pronounced. In fact, the evolution of the thermal patterns depends greatly on the temperature gradient acting on the evaporating droplet. In order to reveal the evolution mechanism of the thermal patterns, the variations of the tangential and normal temperature gradients with the droplet height are depicted in Fig. 5, where the temperature gradients are the average values of at least three sets of data measured by infrared camera. Moreover, the ratio of $M a_{\mathrm{v}}$ to $M a_{\mathrm{h}}$ is also shown in Fig. 5, where $M a_{\mathrm{v}}$ and $M a_{\mathrm{h}}$ represents the Marangoni numbers with respect to the normal and tangential temperature gradients, respectively. They are defined as [29]

$$
\begin{aligned}
& M a_{\mathrm{v}}=\left|\sigma_{T}\right| h \Delta T /(\mu a) \\
& M a_{\mathrm{h}}=\left|\sigma_{T}\right| h^{2} \frac{\partial T}{\partial r} /(\mu a)
\end{aligned}
$$

where $\sigma_{\mathrm{T}}$ is the temperature coefficient of surface tension, $\mu$ is dynamic viscosity, $a$ is the thermal diffusivity, $\Delta T$ is the temper- 


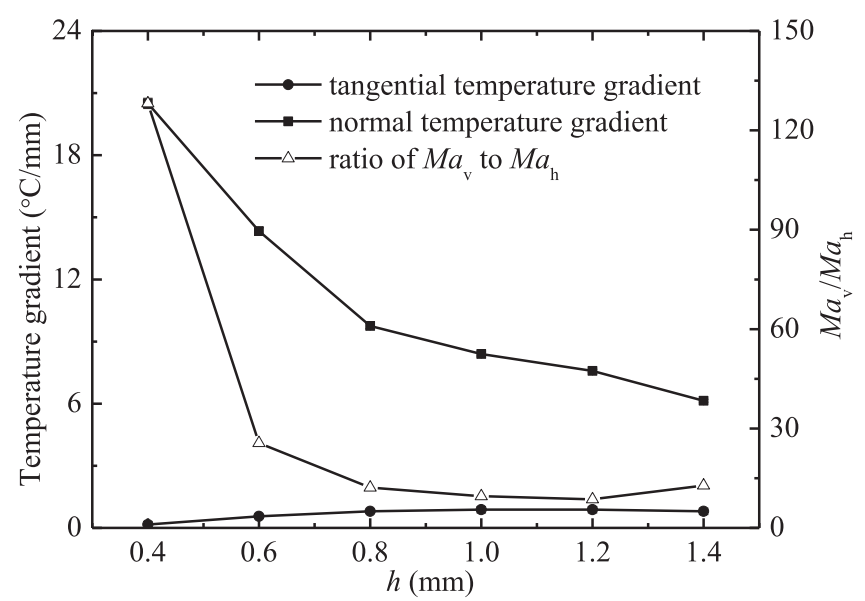

Fig. 5. The tangential and normal temperature gradients, and the ratio of $M a_{\mathrm{v}}$ to $M a_{\mathrm{h}}$ at $T_{\mathrm{w}}=45^{\circ} \mathrm{C}$. ature difference between the bottom and the apex of the droplet, and $\partial T / \partial r$ is the averaged tangential temperature gradient along the droplet surface between $0.5 R$ and $1.0 R$.

As shown in Fig. 5, with the decrease of the droplet height, the tangential temperature gradient tends to decrease, but the normal temperature gradient increases. This means that the effect of tangential temperature gradient weakens, while the effect of the normal temperature gradient increases significantly as the droplet height decreases. In present work, there are two types of thermal patterns. The first type is HTWs characterized by curved stripes that randomly propagate along the longitudinal direction, which is induced by the tangential temperature gradient. The second type is B-M cells characterized by closed cell patterns, which is induced by normal temperature gradient. Actually, according to the definition of $M a_{\mathrm{v}}$ and $M a_{\mathrm{h}}$, the ratio of $M a_{\mathrm{v}}$ to $M a_{\mathrm{h}}$ reflects the relative magnitude of the normal and the tangential temperature gradient effects. When $M a_{\mathrm{v}} / M a_{\mathrm{h}}$ is small, the tangential temperature gradient can induce the HTWs. However, when it is large enough, the effect

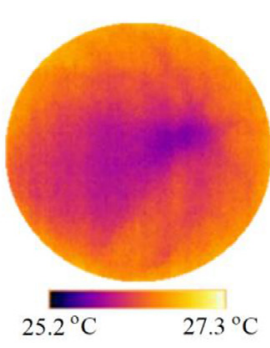

$h=1.2 \mathrm{~mm}$

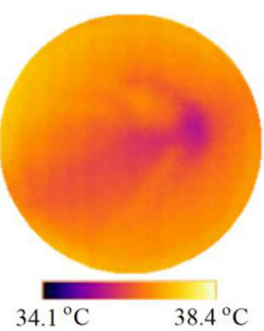

$h=1.2 \mathrm{~mm}$

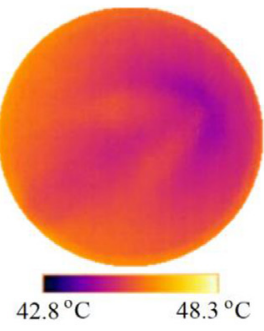

$h=1.2 \mathrm{~mm}$
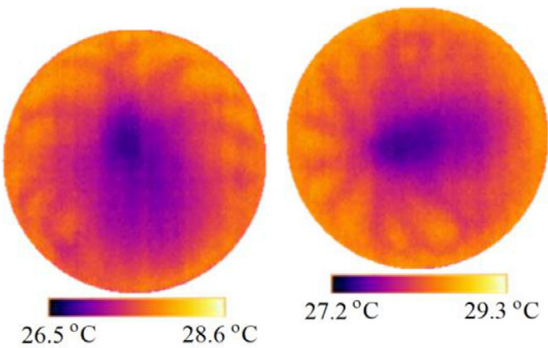

$h=0.6 \mathrm{~mm}$

$h=0.4 \mathrm{~mm}$

(a) $T_{\mathrm{w}}=30^{\circ} \mathrm{C}$
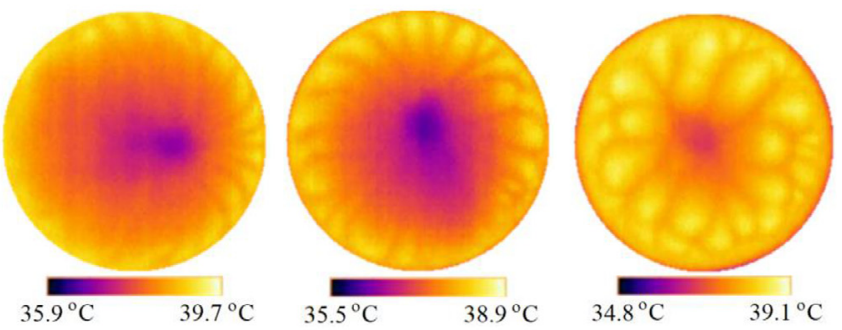

$h=0.4 \mathrm{~mm}$

(b) $T_{\mathrm{w}}=45^{\circ} \mathrm{C}$
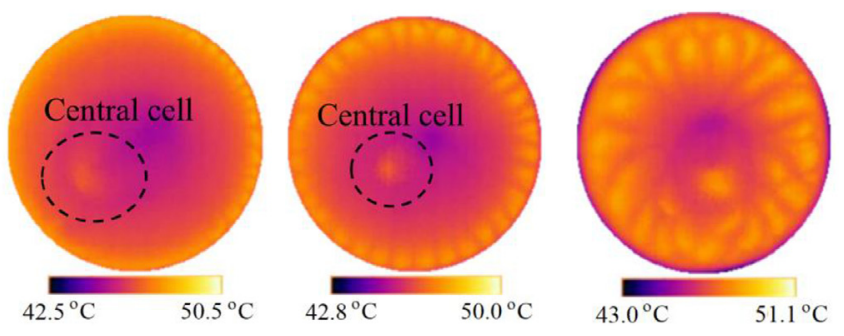

$h=0.4 \mathrm{~mm}$

(c) $T_{\mathrm{w}}=600^{\circ} \mathrm{C}$

Fig. 6. The evolution of thermal patterns with the droplet height at different substrate temperatures. 

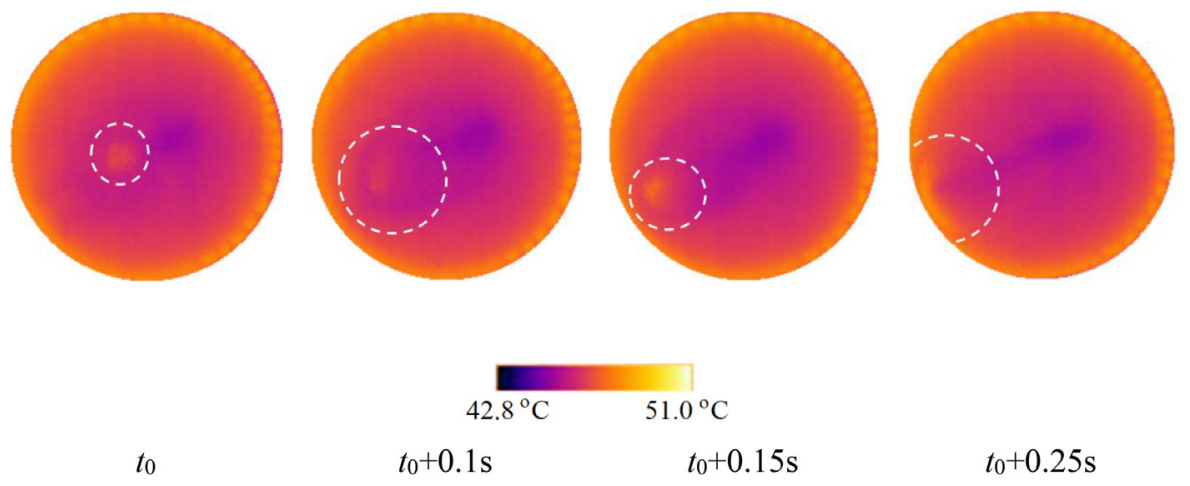

(a) The evolution of central cell.

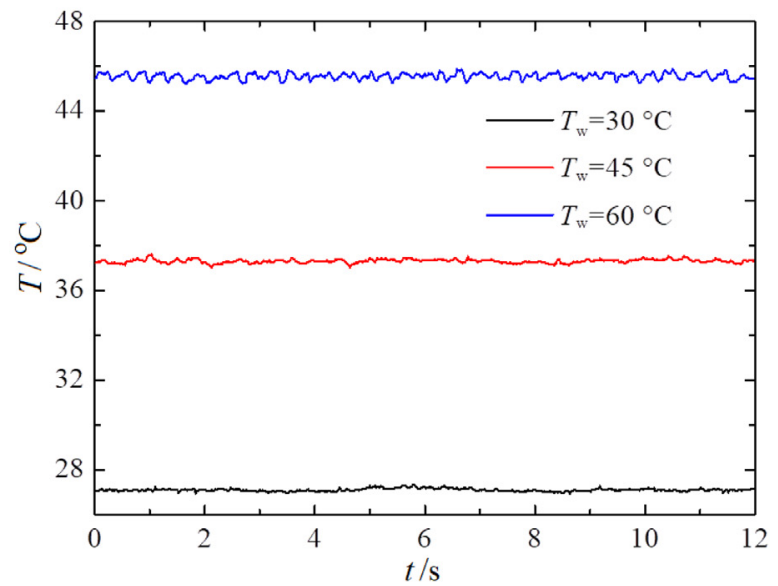

(b) Temperature fluctuation at droplet apex.

Fig. 7. The evolution (a) of the central cell during a period at $T_{\mathrm{w}}=60{ }^{\circ} \mathrm{C}$ and the temperature fluctuations at the droplet apex of the ethanol droplet at $h=0.8 \mathrm{~mm}$.

of the normal temperature gradient is dominant. At this time, B$\mathrm{M}$ cells will appear. The value of $M a_{\mathrm{v}} / M a_{\mathrm{h}}$ increases with the decrease of droplet height. Especially for the case that the droplet height is low enough, the value of $M a_{\mathrm{v}} / M a_{\mathrm{h}}$ becomes very large. For example, when $h=0.4 \mathrm{~mm}, M a_{\mathrm{v}} / M a_{\mathrm{h}} \approx 128$, which means that the Marangoni convection becomes very significant. It is precisely because of the change in the relative size of the tangential and the normal temperature gradients that the thermal pattern changes from HTWs to B-M cells.

In addition to the droplet geometry, the substrate temperature is another important factor affecting the thermal patterns [18,28]. Fig. 6 shows the evolution of the thermal patterns with the droplet height at three different substrate temperature. It can be found that the surface temperature distribution of ethanol droplet shows different characteristics when it evaporates at three different substrate temperatures. In general, the nonuniform temperature distribution near the droplet center, the HTWs and Bénard-Marangoni cells appear in sequence with the decrease of the droplet height. However, it is notably that the surface temperature distribution at $T_{\mathrm{w}}=60{ }^{\circ} \mathrm{C}$ is slightly different from those at $T_{\mathrm{w}}=45^{\circ} \mathrm{C}$ and $30{ }^{\circ} \mathrm{C}$. As marked by black dash circle in Figs. 6(c), an obvious central cell appears at $h=0.8 \mathrm{~mm}$ and $0.6 \mathrm{~mm}$.

Fig. 7 shows the evolution of the central cell at $T_{\mathrm{w}}=60{ }^{\circ} \mathrm{C}$ and the temperature fluctuations at the droplet apex at different substrate temperatures. First, the central cell is generated near the droplet apex, and then it will move towards the contact line and disappear near the contact line, as shown in Fig. 7(a). Interestingly, the evolution of this cell seems to be very periodic. After one cell disappearing near the contact line, another new cell will be generated near the droplet apex. From the temperature fluctuation curve at the droplet apex, it can be found that the temperature fluctuation is very regular at $T_{\mathrm{w}}=60{ }^{\circ} \mathrm{C}$, while there is almost no fluctuation at $T_{\mathrm{w}}=45$ and $30{ }^{\circ} \mathrm{C}$, as shown in Fig. 7(b). According to the temperature fluctuation curve, the periodicity of the central cell appearance is very obvious, which is similar to the results of our previous work [30] at low pressures. When the ethanol droplet evaporates at low pressures, the evaporation rate increases greatly. Therefore, the enhancing evaporative cooling effect leads to the increase of the temperature difference between the vapor-liquid interface and the substrate. As a result, a large normal temperature gradient induces the appearance of the central flow cells. In present work, when the substrate temperature increases to $T_{\mathrm{w}}=60$ ${ }^{\circ} \mathrm{C}$, the evaporation is also very intense, and should be mainly affected by normal temperature gradient. That's why the central cell appears.

Fig. 8 shows the variation of the ratio of $M a_{\mathrm{v}}$ to $M a_{\mathrm{h}}$ with the droplet height when the ethanol droplet evaporates at different substrate temperatures. It can be found that the value of $M a_{\mathrm{v}} / M a_{\mathrm{h}}$ at $T_{\mathrm{w}}=60{ }^{\circ} \mathrm{C}$ is always greater than those at $T_{\mathrm{W}}=45$ and $30{ }^{\circ} \mathrm{C}$. It hints that the Marangoni convection at $T_{\mathrm{w}}=60{ }^{\circ} \mathrm{C}$ is stronger than that at $T_{\mathrm{w}}=45$ and $30{ }^{\circ} \mathrm{C}$ under the same droplet height, which is the reason for the central cell appearance at $T_{\mathrm{W}}=60{ }^{\circ} \mathrm{C}$. It should be noted that the value of $M a_{\mathrm{v}} / M a_{\mathrm{h}}$ at $T_{\mathrm{w}}=45^{\circ} \mathrm{C}$ and $30^{\circ} \mathrm{C}$ and $h=0.6 \mathrm{~mm}$ is larger than one at $T_{\mathrm{w}}=60{ }^{\circ} \mathrm{C}$ and $h=0.8 \mathrm{~mm}$. 


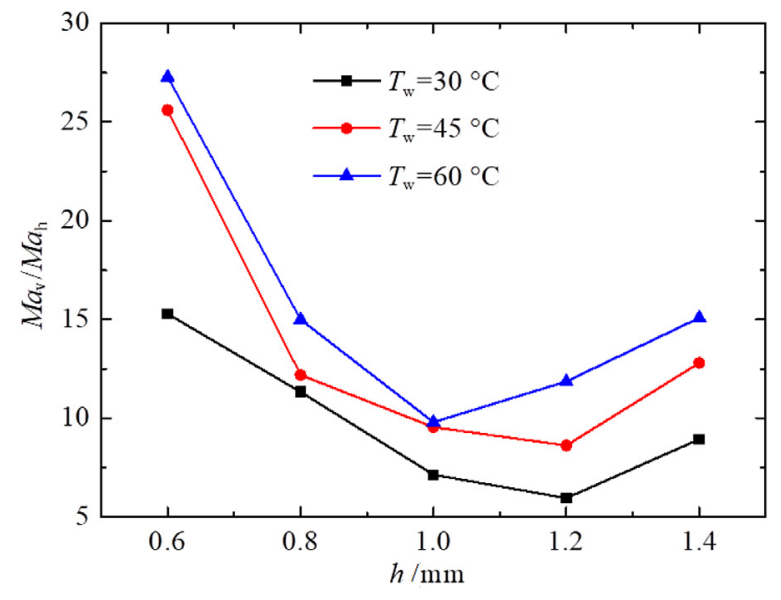

Fig. 8. The variation of the ratio of $M a_{\mathrm{v}}$ to $M a_{\mathrm{h}}$ with the droplet height at different substrate temperatures.

However, $M a_{\mathrm{v}}$ is 14,000 at $T_{\mathrm{w}}=60{ }^{\circ} \mathrm{C}$ and $h=0.8 \mathrm{~mm}$, which is much larger than 6271 at $T_{\mathrm{w}}=45{ }^{\circ} \mathrm{C}$ and 1604 at $T_{\mathrm{w}}=30{ }^{\circ} \mathrm{C}$ at $h=0.6 \mathrm{~mm}$. It is the reason why the central cell does not appear at $T_{\mathrm{w}}=45^{\circ} \mathrm{C}$ and $30^{\circ} \mathrm{C}$ and $h=0.6 \mathrm{~mm}$.

From Fig. 6, it can be seen that the wave number of HTWs increases with the increase of the substrate temperature at $h=0.6 \mathrm{~mm}$. For example, the wave number of HTWs at $T_{\mathrm{W}}=60$ ${ }^{\circ} \mathrm{C}$ is about 33 , while they are 21 and 10 at $T_{\mathrm{w}}=45$ and $30{ }^{\circ} \mathrm{C}$, respectively. This result is in accordance with the previous researches $[18,28,29,32]$. Fig. 9 shows the position of the temperature monitoring point and corresponding temperature fluctuations at different substrate temperatures. It can be found that the frequency

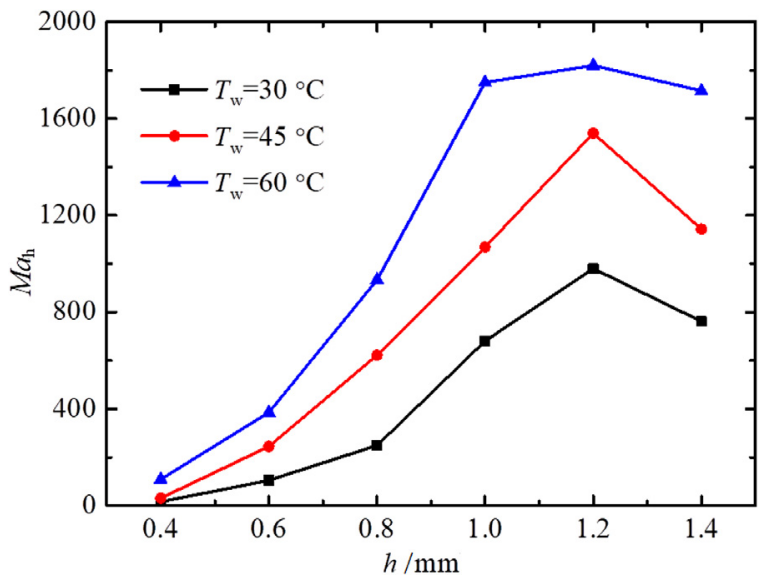

Fig. 10. The variation of $M a_{\mathrm{h}}$ with the droplet height and substrate temperature.

of temperature fluctuation increases with the increase of the substrate temperature.

Fig. 10 exhibits the variation of $M a_{\mathrm{h}}$ with the droplet height and substrate temperature. $M a_{\mathrm{h}}$ increases with the increase of the substrate temperature, which indicates that the thermocapillary convection is gradually enhanced. It is precisely because of the enhancement of thermocapillary convection that both the wave number of HTWs and the frequency of temperature fluctuation increase at a high substrate temperature.

Fig. 11 shows the variation of the evaporation rate $E_{\mathrm{V}}$ with the droplet height and the substrate temperature. Each data point is the average value of the evaporation rate obtained from at

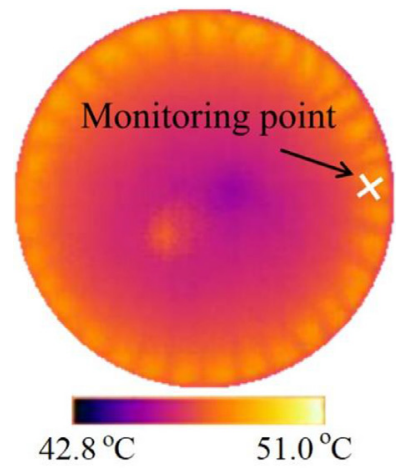

(a) The position of monitoring point

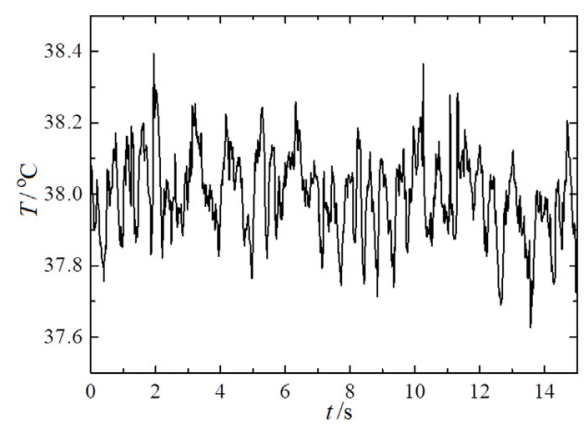

(c) $T_{\mathrm{w}}=45^{\circ} \mathrm{C}$

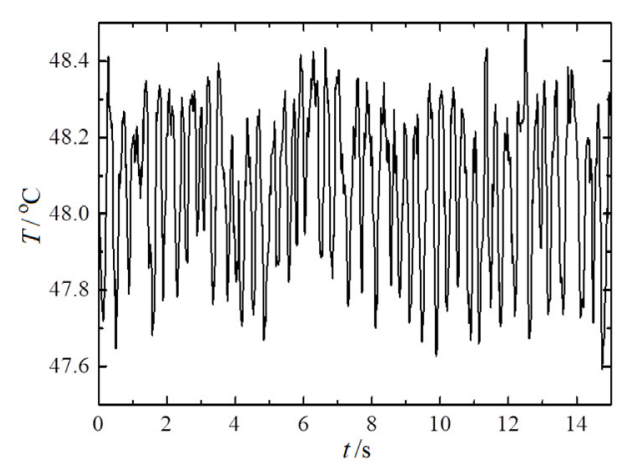

(b) $T_{\mathrm{w}}=60^{\circ} \mathrm{C}$

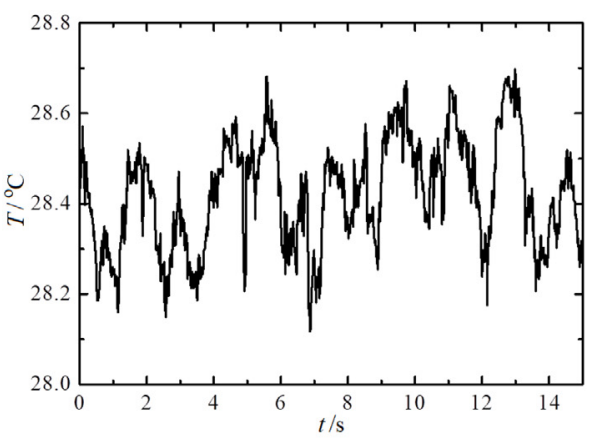

(d) $T_{\mathrm{w}}=30^{\circ} \mathrm{C}$

Fig. 9. The position (a) of the temperature monitoring point and corresponding temperature fluctuations (b-d) at different substrate temperatures at $h=0.6 \mathrm{~mm}$. 


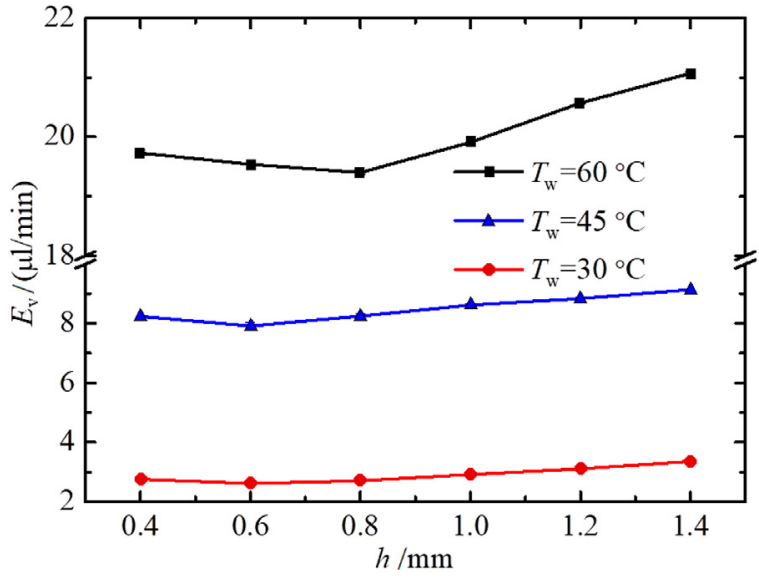

Fig. 11. The evolution of $E_{\mathrm{v}}$ with the change of droplet height and substrate temperature.

least three independent repeated experiments. With the increase of the substrate temperature, the evaporation pressure raises and the ethanol droplet can obtain more energy from the substrate. Therefore, the evaporation rate increases gradually. Furthermore, the evaporation rate decreases first, and then increases with the decrease of the droplet height regardless of the substrate temperature.

In present work, the evaporation rate is mainly affected by the substrate temperature, the droplet surface area and the thermal convection. When the droplet height decreases, the droplet surface area decreases, which will lead to a reduction in the evaporation rate. However, the evaporating interface is closer to the substrate, which means the thermal conduction path becomes shorter. Thus, the surface temperature tends to raise, which will lead to the increase of the evaporation rate.

Moreover, when the evaporating interface is closer to the substrate, the vertical temperature gradient increases and the BénardMarangoni convection is enhanced. As shown in Fig. 5, when the droplet height is equal to $0.4 \mathrm{~mm}$, the entire droplet surface is occupied by Bénard-Marangoni cells. As mentioned above, BénardMarangoni cells are generated near the droplet center or the threephase contact line and move along the latitudinal direction. Therefore, the Bénard-Marangoni convection will carry the warm fluid from near the high-temperature substrate to the evaporating surface. After that, the surface temperature is raised by tangential motion. It is worth mentioning that although the increase in surface temperature reduces the normal temperature gradient and weakens the Bénard-Marangoni convection, under the combined action of the evaporative cooling effect and the normal temperature gradient, the stable Bénard-Marangoni convection is finally formed. Compared with the case where there is no Bénard-Marangoni convection, the surface temperatures of the droplets with BénardMarangoni convection are higher, which promote the evaporation rate. Finally, under the combined effect of the reduction of the evaporating surface area, the raise of the surface temperature and the enhancement of the thermal convection, the evaporation rate tends to decrease first, and then increase with the decrease of the droplet height. It should be noted that the inflection point of the evaporation rate variation occurs earlier because of the presence of central cells at $T_{\mathrm{w}}=60{ }^{\circ} \mathrm{C}$. As can be seen in Fig. 5(c), when the droplet evaporates at $T_{\mathrm{w}}=60^{\circ} \mathrm{C}$, a central cells appears at $h=0.8 \mathrm{~mm}$. It is the central flow cells that take the warm liquid near the substrate to the evaporating surface to promote the evaporation. This phenomenon proves the promotion effect of upward thermal flow (i.e. Bénard-Marangoni cells and central cells) on the evaporation.

\section{Conclusions}

This paper presented a series of the experimental results on the evaporation dynamics of the ethanol droplet on a heated substrate. The evolution of thermal patterns and the variation of the evaporation rate with the droplet height and the substrate temperature are obtained. The main conclusions can be summarized as follows.

(1) No matter how the substrate temperature changes, the temperature distribution near the droplet center is nonuniform fluctuation, and the HTWs and Bénard-Marangoni cells appear in sequence on the evaporating surface with the decrease of the droplet height. The effect of liquid supplementation on the thermal patterns is negligible.

(2) With the raise of the substrate temperature, the tangential and normal temperature gradients increase. Therefore, the thermal convection is enhanced, which results in the increase of both the wave number of HTWs and the temperature fluctuation frequency. Especially, there is an obvious and regular central cell at $T_{\mathrm{w}}=60^{\circ} \mathrm{C}$.

(3) With the decrease of the droplet height, the evaporation rate decreases first, and then increases. The enhancing thermal convection can promote the evaporation process of the ethanol droplet.

\section{Declaration of Competing Interest}

The authors declared that there is no conflict of interest.

\section{Acknowledgement}

This work is supported by the National Natural Science Foundation of China (Grant No. 11532015, 51776022) and Science and Technology Research Project of Chongqing Education Commission (Grant No. KJQN201903305).

\section{Supplementary materials}

Supplementary material associated with this article can be found, in the online version, at doi:10.1016/j.ijheatmasstransfer. 2020.120352.

\section{References}

[1] J. Park, J. Moon, Control of colloidal particle deposit patterns within picoliter droplets ejected by ink-jet printing, Langmuir 22 (8) (2006) 3506-3513.

[2] M. Ren, J. Sweelssen, N. Grossiord, H. Gorter, T.M. Eggenhuisen, R. Andriessen, Inkjet printing technology for OPV applications, J. Imaging Sci. Technol. 56 (4) (2012) 1-5.

[3] P. Bhattacharya, A. Samanta, S. Chakraborty, Spray evaporative cooling to achieve ultra fast cooling in runout table, Intern. J. Thermal Sci. 48 (9) (2009) $1741-1747$.

[4] J. Kim, Spray cooling heat transfer: the state of the art, Int. J. Heat Fluid Flow 28 (4) (2007) 753-767

[5] S. Paria, R.G. Chaudhuri, N.N. Jason, Self-assembly of colloidal sulfur particles on a glass surface from evaporating sessile drops: influence of different salts New J. Chem. 38 (12) (2014) 5943-5951.

[6] Y.-.K. Kim, H.-.K. Na, S. Ham, D.-.H. Min, Mediating ordered assembly of gold nanorods by controlling droplet evaporation modes for surface enhanced Raman scattering, RSC Adv 4 (91) (2014) 50091-50096.

[7] H. Hu, R.G. Larson, Marangoni effect reverses coffee-ring depositions, J. Phys. Chem. B 110 (14) (2006) 7090-7094

[8] R. Malinowski, G. Volpe, I.P. Parkin, G. Volpe, Dynamic control of particle deposition in evaporating droplets by an external point source of vapor, J. Phys. Chem. Lett. 9 (3) (2018) 659-664.

[9] M.A. Mahmud, B.D. MacDonald, Experimental investigation of interfacial energy transport in an evaporating sessile droplet for evaporative cooling applications, Phys. Rev. E 95 (1) (2017) 012609.

[10] H. Ghasemi, C. Ward, Energy transport by thermocapillary convection during sessile-water-droplet evaporation, Phys. Rev. Lett. 105 (13) (2010) 136102.

[11] K. Sefiane, J. Moffat, O. Matar, R. Craster, Self-excited hydrothermal waves in evaporating sessile drops, Appl. Phys. Lett. 93 (7) (2008) 074103. 
[12] D. Mamalis, V. Koutsos, K. Sefiane, Nonisothermal spreading dynamics of self-rewetting droplets, Langmuir 34 (5) (2018) 1916-1931.

[13] X. Chen, X. Wang, P.G. Chen, Q. Liu, Thermal effects of substrate on Marangoni flow in droplet evaporation: response surface and sensitivity analysis, Int. J. Heat Mass Transf. 113 (2017) 354-365.

[14] T. Josyula, Z. Wang, A. Askounis, D. Orejon, S. Harish, Y. Takata, P.S. Mahapatra, A. Pattamatta, Evaporation kinetics of pure water drops: thermal patterns, Marangoni flow, and interfacial temperature difference, Phys. Rev. E 98 (5) (2018) 052804.

[15] K. Gleason, H. Voota, S.A. Putnam, Steady-state droplet evaporation: contact angle influence on the evaporation efficiency, Int. J. Heat Mass Transf. 101 (2016) 418-426.

[16] P. Chen, S. Harmand, S. Ouenzerfi, J. Schiffler, Marangoni Flow Induced Evaporation Enhancement on Binary Sessile Drops, J. Phys. Chem. B 121 (23) (2017) 5824-5834.

[17] S. Semenov, F. Carle, M. Medale, D. Brutin, 3D unsteady computations of evaporative instabilities in a sessile drop of ethanol on a heated substrate, Appl. Phys. Lett. 111 (24) (2017) 241602.

[18] D. Brutin, B. Sobac, F. Rigollet, C. Le Niliot, Infrared visualization of thermal motion inside a sessile drop deposited onto a heated surface, Exp. Therm. Fluid Sci. 35 (3) (2011) 521-530.

[19] K. Sefiane, Y. Fukatani, Y. Takata, J. Kim, Thermal patterns and hydrothermal waves (HTWs) in volatile drops, Langmuir 29 (31) (2013) 9750-9760.

[20] C.A. Ward, F. Duan, Turbulent transition of thermocapillary flow induced by water evaporation, Phys. Rev. E 69 (5 Pt 2) (2004) 056308.

[21] R.G. Picknett, R. Bexon, The evaporation of sessile or pendant drops in still air, J. Colloid Interface Sci. 61 (2) (1977) 336-350.

[22] M.E.R. Shanahan, C. Bourgès, Effects of evaporation on contact angles on polymer surfaces, Int. J. Adhes. Adhes. 14 (3) (1994) 201-205.
[23] S.M.M. Ramos, J.F. Dias, B. Canut, Drop evaporation on superhydrophobic PTFE surfaces driven by contact line dynamics, J. Colloid Interface Sci. 440 (2015) 133-139.

[24] M.E.R. Shanahan, Simple Theory of "Stick-Slip" Wetting Hysteresis, Langmuir 11 (3) (1995) 1041-1043.

[25] G. Lu, Y.-.Y. Duan, X.-.D. Wang, D.-.J. Lee, Internal flow in evaporating droplet on heated solid surface, Int. J. Heat Mass Transf. 54 (19-20) (2011) 4437-4447.

[26] C. Bouchenna, M.A. Saada, S. Chikh, L. Tadrist, Investigation of Thermo-Capillary Flow Inside an Evaporating Pinned Water Droplet, Interfacial Phenom. Heat Transf. 3 (2) (2015) 185-201.

[27] X. Zhong, F. Duan, Stable hydrothermal waves at steady state evaporating droplet surface, Sci. Rep. 7 (1) (2017) 1-9.

[28] S. Ye, C.-.M. Wu, L. Zhang, Y.-.R. Li, Q.-.S. Liu, Evolution of thermal patterns during steady state evaporation of sessile droplets, Exp. Therm. Fluid Sci. 98 (2018) 712-718.

[29] T.-.S. Wang, W.-.Y. Shi, Transition of Marangoni convection instability patterns during evaporation of sessile droplet at constant contact line mode, Int. J. Heat Mass Transf. 148 (2020) 119138.

[30] S. Ye, L. Zhang, C.-.M. Wu, Y.-.R. Li, Q.-.S. Liu, Experimental investigation of evaporation dynamic of sessile droplets in pure vapor environment with low pressures, Int. J. Thermal Sci. 149 (2020) 106213.

[31] M. Pack, H. Hu, D.-.O. Kim, X. Yang, Y. Sun, Colloidal Drop Deposition on Porous Substrates: competition among Particle Motion, Evaporation, and Infiltration, Langmuir 31 (29) (2015) 7953.

[32] K. Sefiane, A. Steinchen, R. Moffat, On hydrothermal waves observed during evaporation of sessile droplets, Colloid Surf. A 365 (1-3) (2010) 95-108.

[33] R. Tuckermann, S. Bauerecker, H. Cammenga, IR-thermography of evaporating acoustically levitated drops, Int. J. Thermophys. 26 (5) (2005) 1583-1594. 